\section{The Meson Spectrum Near Sea-Level}

L. Janossy and J. C. Wilson determined, some time ago ${ }^{1}$, from a comparison of theoretical and experimental spectra at sea-level the exponent $\gamma$ in the production spectrum of mesons $d N=$ const. $p^{-\gamma d p}$; they find a value $\gamma=3 \cdot 3$. As this value differs appreciably from the ordinarily accepted one, we have thought it worth while to check the above result by a more accurate method, taking account of the variable energy-loss of mesons through the atmosphere.

The basis of our computation is a formula for the vertical meson spectrum at sea-level derived by M. E. Rose ${ }^{2}$ with the hypothesis of an initial $\varepsilon^{-\gamma}$ spectrum. This formula is

$$
f\left(\varepsilon, t_{s}\right) d \varepsilon=C_{0} \frac{B\left(\varepsilon^{\prime}\right)}{B(\varepsilon)} \varepsilon^{\prime}-\gamma \exp (-\alpha D) d \varepsilon,
$$

where $\varepsilon^{\prime}$ is the energy at the production level of a meson having energy $\varepsilon$ at sea-level ; $B(\varepsilon)$ is the energy loss $-\frac{d \varepsilon}{d t}\left(t\right.$ in gm./cm. $\left.{ }^{2}\right) ; \alpha=\frac{t_{s}}{p_{s} c \tau_{0}}$ (with $t_{s}$ height of the atmosphere in gm./cm. ${ }^{2}$, $p_{s}$ air density at sealevel, $c=3 \times 10^{10} \mathrm{~cm}$. $/$ sec., and $\tau_{0}$ mean life of the meson at rest); $D$ is the integral $\int_{P_{8}}^{R_{0}} \frac{d R}{t p(\bar{R})}$, with $R_{0}$, $R_{s}$ residual ranges (in $\mathrm{gm} . / \mathrm{cm} .^{2}$ ) of mesons of energies $\varepsilon^{\prime}$ and $\varepsilon$ ( $p$ momentum of the meson).

Rose's expression is quite different from that given by Euler and Heisenberg ${ }^{3}$, owing to a different treatment of the absorption of mesons. However, it is easily verified that it satisfies the following differential equation :

$$
\frac{\partial f}{\partial t}=B(\varepsilon) \frac{\partial f}{\partial \varepsilon}+f \frac{d B(\varepsilon)}{d \varepsilon}-f \frac{\alpha}{p t},
$$

where $f(\varepsilon, t)$ is the differential energy spectrum at level $t$. This equation is the generalization of that of Euler and Heisenberg to the case of variable energy loss (and energy- instead of momentum-distribution).

Assuming an initial spectrum $p^{-\gamma}$, instead of $\varepsilon^{-\gamma}$, Rose's formula becomes

$d N=C \frac{B\left(\varepsilon^{\prime}\right)}{B(\varepsilon)}\left(\varepsilon^{\prime 2}+2 \varepsilon^{\prime}\right)^{-(\gamma+1) / 2}(\varepsilon+1) \exp (-\alpha D) d \varepsilon$.

(Energy unit $=\mu c^{2}=\sim 10^{8} \mathrm{eV}$.)

We have used this formula. It contains three parameters to be adjusted from experimental data: the normalization factor $C$, the exponent $\gamma$ and $\alpha$ (corresponding to the mean life $\tau_{0}$ of the meson). Each of these parameters appears linearly in the expression of $\log d N / d \varepsilon$, making it relatively easy to apply a least squares method to $\log d N / d \varepsilon$ and determine the parameters simultaneously.

The experimental data were those used by Jánossy and Wilson, namely, those of Blackett, Jones and Wilson, with the exclusion of the doubtful value near $\varepsilon=1.5 \times 10^{10} \mathrm{eV}$. To avoid giving a greater statistical weight to the high-energy region owing to the greater number of determinations, we averaged the experimental data, taking as experimental spectrum (normalized to maximum value 1):

$\begin{array}{lccc}\varepsilon & \frac{d N}{d \varepsilon} & \varepsilon & \frac{d N}{d \varepsilon} \\ 0.8 & 0.40 & 14.03 & 0.81 \\ 1.69 & 0.81 & 24.02 & 0.49 \\ 3.13 & 0.83 & 44.01 & 0.195 \\ 6.57 & 1.00 & 79.0 & 0.061\end{array}$

Application of the least squares method gives $\gamma=3 \cdot 20, \tau_{0}=1.69 \times 10^{-6}$ sec., $C=2 \cdot 67 \times 10^{5}$.

This seems to confirm that $\gamma$ has a value somewhat higher than that usually accepted. The value of $\tau_{0}$ seems rather small in comparison with the best experimental data, and it would have been even smaller had we used an initial $\varepsilon^{-\gamma}$ spectrum. It would be difficult, however, to draw any inference from this fact, owing to the uncertainty which still exists in the low-energy part of the spectrum.

The effect of a departure $\theta$ of the measured meson tracks from the vertical direction was also considered, but it was found that with a distribution of the form const. $(l \cos \theta-r \sin \theta)$ valid for parallel counters of length $l \mathrm{~cm}$., placed $r \mathrm{~cm}$. apart from one another, no important errors should occur up to a maximum value of $30^{\circ}$ for $\theta$, which appears to be a reasonable upper limit.

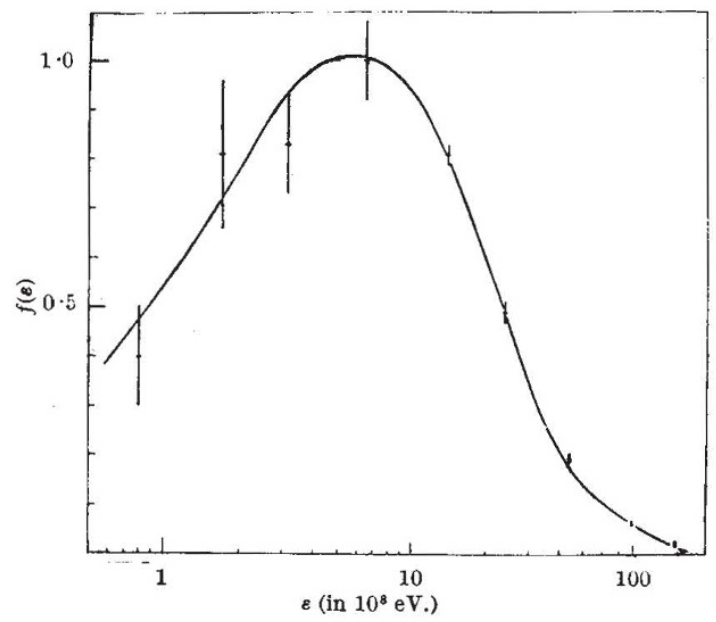

The graph shows the accord between experimental data and theoretical curve.

$$
\begin{aligned}
& \text { M. DELla Corte } \\
& \text { T. F. FAzzini }
\end{aligned}
$$

S. A. Franchetti

Physical Institute,

University of Florence. March 25.

' Nature, 158, 450 (1946).

${ }^{2} J$. Franklin Inst., 236, 9 (1943).

${ }^{3}$ Ergeb. Exak. Naturwiss., 40 (1938).

\section{Diffuse Bands on Laue Photographs of Silver Chloride Crystals}

IN a recent paper and in a communication in Nature $^{1}$, Laue photographs of large silver chloride crystals were published, showing diffuse bands and spots. It was then suggested that these bands were, at least partly, not of thermal origin, but due to the presence of definite lattice defects, parallel to the cubic planes and axes. The experimental fact that such crystals, when irradiated with ultra-violet light, showed an 'optical etching effect' parallel to the cubic planes (as evident from the reflexion of incident light by the irradiated crystals, which was similar to that shown by chemically etched crystals) was considered to support this conclusion. Moreover, on a photograph of a crystal cooled with liquid air, taken at the time the above paper was published, the diffuse pattern was still visible. 